\title{
A Proposal of the Person-centered Approach for Personal Task Management
}

\author{
Ikki Ohmukai \\ The Graduate University for Advanced Studies \\ Department of Informatics \\ 2-1-2 Hitotsubashi, Chiyoda-ku, Tokyo, Japan \\ i2k@grad.nii.ac.jp
}

\author{
Hideaki Takeda \\ National Institute of Informatics \\ 2-1-2 Hitotsubashi, Chiyoda-ku, Tokyo, Japan \\ takeda@nii.ac.jp
}

\author{
Mitsunori Miki \\ Doshisha University \\ 1-3 Tatara-miyakodani, Kyotanabe-shi, Kyoto, Japan \\ mmiki@mail.doshisha.ac.jp
}

\begin{abstract}
This paper proposes a person-centered approach for personal task management in which people can decide management of their tasks according to their environments including their subjective and multivalent judgement and human relationship. In our approach the task management is modeled as a decision-making process on their own resources. Human decision-making process consists of three types of activity, i.e., the intelligence activity, the design activity, and the choice activity. The proposal system assists each activity by three sub-systems, i.e., Visualizer, Optimizer, and Recommender respectively. At first, Visualizer indicates the attributes associated with each task such as the deadline, the subjective priority, and the workload, which are determined by the user. And Optimizer generates executable schedules from these tasks by active scheduler and multi-objective genetic algorithm. Finally, Recommender evaluates these alternatives by analytic hierarchy process. Also the system is able to analyze the human relationship of the user group with PageRank algorithm, and this result is utilized to improve the performance of the task scheduler. We implement client/server system which uses mobile phones. And we verify the function of the proposed system along the lines of two scenarios.
\end{abstract}

\section{Introduction}

In this paper, we propose a person-centered approach for personal task management in which people can treat with their tasks appropriately according to their environments including their subjective and multivalent judgement and human relationship.
The features of our approach as task management are not only to assist scheduling tasks but also to assist people to clarify their intension for tasks, e.g., awareness of inconsistency and subjective judgements for tasks.

In this approach the task management is modeled as a decision-making process[10] on their own resources. As we design our personal task management system with the framework of decision-making theory, it consists of three basic processes that corresponds to three types of activities in decision-making theory.

Human decision-making process consists of three types of activity, i.e., the intelligence activity, the design activity, and the choice activity. The proposed system assists these activities with Visualizer, Optimizer, and Recommender respectively.

Firstly, Visualizer indicates the attributes associated with each task such as the deadline, the subjective priority, and the workload, which are determined by the user. Secondly, Optimizer generates executable schedules from these tasks by optimization algorithms. Thirdly, Recommender evaluates these alternatives by an analytic process. The system is also able to analyze the human relationship of the user group, and this result is utilized to improve the performance of the task scheduler.

We show how the proposed system can work with two scenarios. In the first scenario, we show how to resolve overlap of tasks. In the second, we establish the process that the priority of client is calculated and propagated.

This paper organizes as follows. In Section 2, we describe the personal task scheduling problem and clarify the difficulty of this problem. In Section 3, we propose a new approach based on decision-making theory. We explain three types of activities of human decision process and redefine the personal task schedule problem coordinating with 
these processes. In Section 4, we describe the proposed system in detail. We show functions of Visualizer, Optimizer, Recommender, and Analyzer. In Section 5, we confirm the functions of the proposed system along with two scenarios. We describe the specification of the implemented system in Section 6, we summarize the paper in the last section.

\section{Personal Task Schedule Management}

In this section, we clarify our objective problems.

\subsection{Personal Resource Management}

Managing our resources such as time and money is important today. Complexity about the personal resource management is increasing along with growth of complexity of our society.

Most people now belong to multiple organizations or communities, and assign most of their resources to achieve their objectives, and they entrust the management to them. In recent years, the social structure is in the process of drastically changing. And such change affects people's lifestyle.

For example, suppose a person who belongs not only a business company but also a non-government organization (NGO) or non-profit organization (NPO), which is playing an important role in our society. In this situation, it is difficult that multiple organizations manage his/her resource cooperatively.

There are many studies on groupware and resource coordination based on software agent[3]. However, these studies aim to support a single organization.

\subsection{Personal Task-scheduling Problem}

Since tasks for individuals have variety in their purpose and value, it is difficult to provide a single definitive objective function. We have a number of tasks at any time, and should decide among these tasks which is to perform. Each task has some attributes, such as the deadline, the subjective priority, and the workload. People have their own standards for judging. It makes the evaluation process complicated.

For example, one may prefer to perform smaller and easier tasks at first, then she/he might fail to perform important tasks because they tend to be bigger and more difficult. For improvement in personal productivity, it is important to evaluate tasks from a multifaceted and a long-term perspective. Conventional scheduling tools such as the day planner and the calendar are not adequate to manage the tasks properly.

There are some issues about this problem.

- Objectives and Constraints
Most of the ordinary scheduling problems assume that all of the given tasks must be assigned to one's schedule. However, it is not necessary to perform all tasks in the personal task-scheduling problem. The proposed system deals with this problem as a multi-objective optimization problem. Thus alternatives obtained by the system may not contain all of the given tasks.

\section{- Type of task}

We define two types of task, one is a movable task which can be assigned whenever possible. The other is a fixed task which cannot change their time such as meetings or conferences. There are few studies on the scheduling problem with mixed task environment.

- Dynamically changes of environment

The tasks and their objectives may change when new tasks come in.

\section{Task Management as Decision Support System}

To solve problems described in the previous section, we apply a concept of decision-making theory and decision support systems to them.

Decision support systems provide a better solution for a decision-making entity with interactive operations of data and models.

Decision-making is not a one-shot action such a determination or selection. Simon argued that human decisionmaking process consists of three types of activity, i.e.: the intelligence activity, the design activity, and the choice activity [10]. Consequently, the decision support system should assist these three activities.

Conventional decision support systems tried to resolve problems of which objective is complicated but welldefined. To design the systems for these problems, the designer defines objective functions that mean which state is the best, then she/he describes relations between the objective functions and each of decision-making process. These systems can evaluate a relevance of alternatives and decide the best solutions in them. However this method is applicable only if the designer can define suitable objective functions.

Consequently we formulate the personal task-scheduling problem as an iterative process, i.e.,

1) The system generates several active schedules for a set of tasks.

2) The user selects one of the schedules, and executes it.

3 ) Return to 1) when a new task comes in or an unforeseen incident occurs.

And the system applies support algorithms continuously to each process. 


\section{The Proposed System}

We show the configuration of the proposed system as shown in fig.1. The system is based on server/client architecture. The server has a task database and provides four features as described later. Mobile phones are used to access to the system.

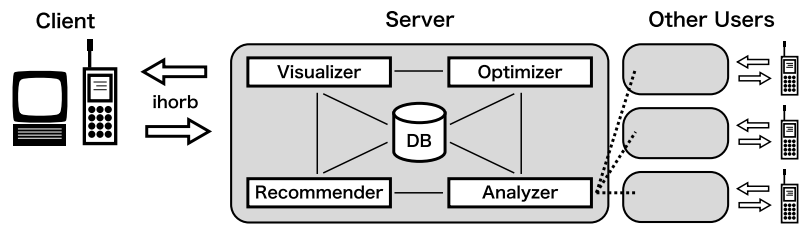

Figure 1. System configuration

\subsection{Data Structure}

The user of the system enters task information with the following attributes:

- Name of task

- Time parameters

- Start time

- Deadline

- Workload

The movable and the fixed task are distinguishable whether these parameters are defined or not.

\section{- Priority}

Decision maker evaluates each task subjectively on scale of 1 to 5 .

\section{- Client}

\subsection{Visualizer: Support for Intelligence Activity}

The process that a user recognizes status of her/his own task scheduling will create an opportunity for a better management. Therefore it is important that the decision support systems extract issues related to task scheduling from the given data.

Since the mobile phones have only small screens and cannot display high-resolution graphics, Visualizer can show the chronogram by different scale, i.e., weekly and monthly. This method stimulates awareness of users' state of task. It shows users not only how many tasks they have, but also overlap of tasks in an easy-to-understand way.

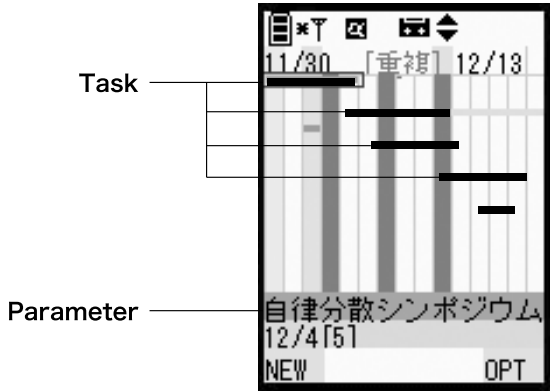

Figure 2. 2D interface on prototype system

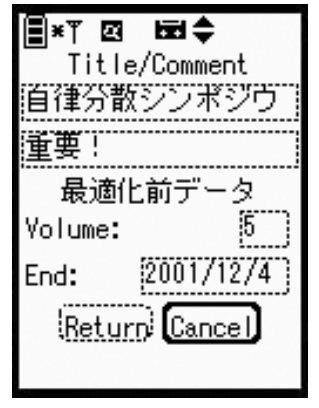

Figure 3. Task parameters

Fig. 2 and 3 show how Visualizer works in the prototype system. In the chronogram in fig. 2, each task is displayed as a horizontal bar of which width indicates the workload of the task along the time axis. The attributes of each task can be changed in the dialog box as shown in fig.3.

The Visualizer warns the user when it detects overlapped tasks or an unexecutable task. Then it displays a button that invokes Optimizer if it judges the necessity for rescheduling.

\subsection{Optimizer: Support for Design Activity}

It is important to allocate her/his resource correctly for problem solving. Depending on users' request, the decision support systems generate active schedules.

Active scheduler resolves the overlaps of the given tasks to obtain executable schedules. It serializes the tasks by changing the attributes of each task.

The Optimizer generates alternate schedules if the active scheduler fails to resolve problems about the overlapped tasks. As described in Section 2, criterion of evaluation are often multiple. Consequently, multi-objective optimization algorithms must be used to calculate these schedules. We use the Genetic Algorithm: GA[2], especially the MultiObjective GA: MOGA[1]. Objective functions for MOGA are set out as follows: 
- Maximize the number of task

- Maximize the utility rate of time

- Maximize the reward based on the subjective priority

- Maximize the reward based on the priority of client

MOGA eliminates non-dominant candidates which is inferior to other solutions in all objective functions. The survivors are passed to Recommender as alternatives.

\subsection{Recommender: Support for Intelligence Ac- tivity}

Most of decision-making problems require qualitative information. Therefore conventional methods based on mathematical model cannot deal with these problems well.

We propose a new method based on an Analytic Hierarchy Process: AHP[9], which is able to treat both qualitative data and quantitative data. In common AHP, the number of pairwise comparison increases considerably along with the increase of the alternatives. It is also necessary to redo the pairwise comparison when a new alternate is added. We apply the absolute measurement method[8] to avoid these ploblems.

\subsection{Analyzer: Estimation of Priority of Client}

The Analyzer supports efficient decision-making to the users with new information extracted.

It is considered that there are some attributes of the task changing with clients. Therefore, we propose a method to calculate priority of client with the PageRank algorithm[7].

\section{Task Management Scenarios with Our Method}

In this section, we show the way to use the proposed system.

\subsection{Scenario 1: Presentation of the Schedule and Resolving the Problems}

This scenario describes a scheduling procedure with $\mathrm{Vi}$ sualizer, Optimizer, and Recommender. At the beginning of the scenario, five tasks are registered as the test problem shown in table 1.

Visualizer displays these tasks as fig. 4. A black bar means a movable task, and a white one means a fixed task. It indicates that two overlaps are occurred. The system invokes Optimizer to resolve them. Optimizer sets start time of task $c$ to before the value of workload of task $b$. As a result, the first overlap is resolved.
Table 1. Scenario 1: Test data

\begin{tabular}{c|c|c|l|c|c} 
Name & Start time & Deadline & Workload & Pri. & From \\
\hline \hline A & - & $1 / 1015: 00$ & $10: 00$ & 3 & W \\
B & $1 / 1111: 00$ & $1 / 1115: 00$ & $4: 00$ & 5 & $\mathrm{X}$ \\
$\mathrm{C}$ & - & $1 / 1120: 00$ & $12: 00$ & 4 & $\mathrm{Y}$ \\
$\mathrm{D}$ & - & $1 / 1314: 00$ & $11: 00$ & 2 & $\mathrm{Z}$ \\
$\mathrm{E}$ & - & $1 / 1318: 00$ & $13: 00$ & 5 & $\mathrm{~W}$
\end{tabular}

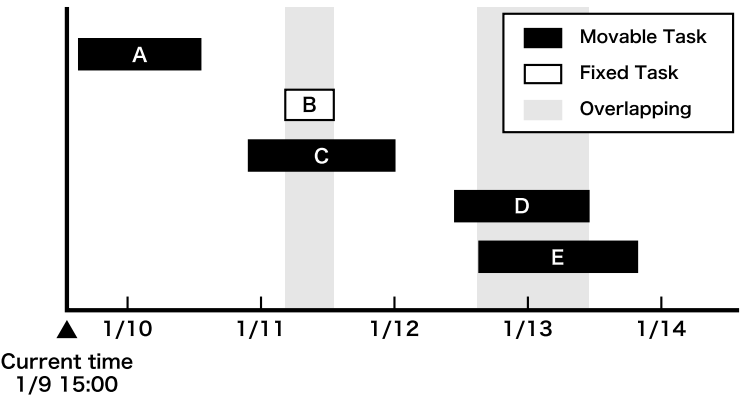

Figure 4. Scenario 1: Displaying initial condition by Visualizer

Next Optimizer moves up task $d$ to resolve the second overlap. But a new overlap between task $c$ and task $d$ is occurred. In this manner, these overlap may take place into a chain reaction. As a consequence, it may be failed to schedule as shown in fig. 5 .

Optimizer generates active schedules by multi-objective genetic algorithm. Table 2 shows six candidates and their evaluation values for four functions. A digit given in parentheses indicates rank of merit by each function. And fig. 6 shows the rank of six alternatives. All of evaluation items of schedule 6 underruns those of schedule 2 or 4 , whereby schedule 6 is eliminated from alternatives.

Recommender evaluates 5 alternatives which are not

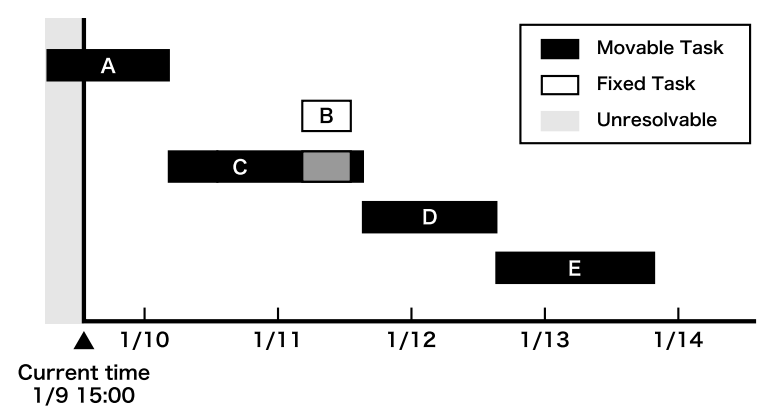

Figure 5. Scenario 1: Fail to schedule 
Table 2. Scenario 1: Evaluation value of alternatives

\begin{tabular}{c|c|c|c|c} 
Alternative & $N_{\text {task }}$ & $R_{\text {util }}$ & $R_{\text {pri. }}$ & $R_{\text {from }}$ \\
\hline \hline Schedule 1 & $5(1)$ & $0.500(6)$ & $19(1)$ & $17(1)$ \\
Schedule 2 & $4(2)$ & $0.851(2)$ & $16(3)$ & $14(3)$ \\
Schedule 3 & $4(2)$ & $0.979(1)$ & $14(5)$ & $12(6)$ \\
Schedule 4 & $4(2)$ & $0.809(4)$ & $15(4)$ & $15(2)$ \\
Schedule 5 & $4(2)$ & $0.830(3)$ & $17(2)$ & $13(5)$ \\
Schedule 6 & $4(2)$ & $0.787(5)$ & $14(5)$ & $14(3)$
\end{tabular}

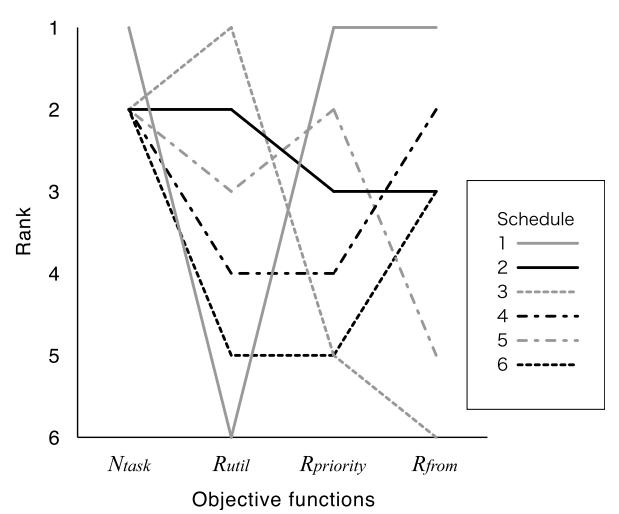

Figure 6. Scenario 1: Rank of alternatives

subject to other candidates. We determine the pairwise comparison matrix as shown in table 3. Table 4 shows the weighting vector of evaluation, that is eigenvector of the pairwise comparison matrix.

Table 5 shows the final result calculated by AHP. Schedule 5 receives the best rate because it has a high evaluation value for $R_{u t i l}$ and $R_{\text {priority }}$ which are major components of its weighting vector.

Table 3. Scenario 1: Matrix for pairwise comparison

\begin{tabular}{c|c|c|c|c} 
& $N_{\text {task }}$ & $R_{\text {util }}$ & $R_{\text {pri. }}$ & $R_{\text {from }}$ \\
\hline \hline$N_{\text {task }}$ & 1 & $1 / 7$ & $1 / 5$ & $1 / 3$ \\
$R_{\text {util }}$ & 7 & 1 & 1 & 3 \\
$R_{\text {priority }}$ & 5 & 1 & 1 & 5 \\
$R_{\text {from }}$ & 3 & $1 / 3$ & $1 / 5$ & 1
\end{tabular}

Table 4. Scenario 1: Weighting vector of criteria

\begin{tabular}{c|c} 
Criterion & Weight \\
\hline \hline$N_{\text {task }}$ & 0.059 \\
$R_{\text {util }}$ & 0.391 \\
$R_{\text {priority }}$ & 0.424 \\
$R_{\text {from }}$ & 0.126
\end{tabular}

Table 5. Scenario 1: Final result

\begin{tabular}{l|c} 
Alternative & Evaluation value \\
\hline \hline Schedule 1 & $0.193(5)$ \\
Schedule 2 & $0.204(2)$ \\
Schedule 3 & $0.202(3)$ \\
Schedule 4 & $0.196(4)$ \\
Schedule 5 & $0.206(1)$
\end{tabular}

\subsection{Scenario 2: Estimation of Priority based on Human Relationship}

In the previous scenario, the priority of client is calculated simply based on frequency of task request shown in fig. 7. However, human relationship of task request is bidirectional and complicated.

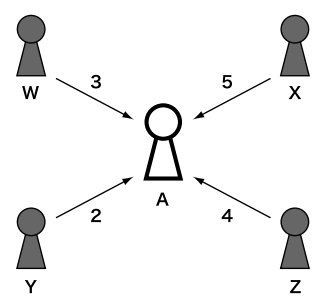

Figure 7. Scenario 2: Dependency relation of task (individual)

In this scenario, a small-scale group is targeted as shown in fig. 8. This group consists of five people, and frequency of task request is shown with the arrows. Analyzer collects these data and compiles into weighted adjacency matrix as shown in fig. 6.

Analyzer then applies PageRank algorithm to this matrix. Table 7 shows the obtained priority, i.e., the eigenvector of the matrix.

The system overwrites the attributes of tasks and reschedules with them. Table 8 shows the recalculated value by Optimizer and table 9 is the final result by Recommender. As compared to table 5, the comprehensive evalu- 


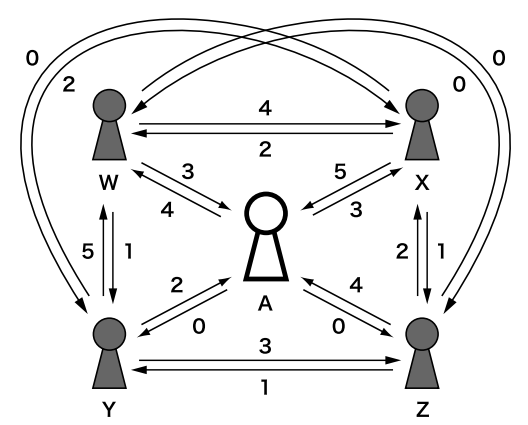

Figure 8. Scenario 2: Dependency relation of task (group)

Table 6. Scenario 2: Task Network

\begin{tabular}{|c||c|c|c|c|c|}
\hline Name & A & W & X & Y & Z \\
\hline \hline A & 0 & 4 & 3 & 0 & 0 \\
\hline W & 3 & 0 & 4 & 1 & 0 \\
\hline X & 5 & 2 & 0 & 0 & 1 \\
\hline Y & 2 & 5 & 2 & 0 & 3 \\
\hline Z & 4 & 0 & 2 & 1 & 0 \\
\hline
\end{tabular}

ation values of schedule 4 and 5 decrease relatively, and the values of schedule 2 and 3 increase because they contain the tasks from $\mathrm{Y}$ and $\mathrm{Z}$ who are evaluated highly by Analyzer.

As just described, the analyzer makes the user's state apparently and changes a suggestion of the system.

\section{Implimentation}

We developed a prototype system based on the personcentered approach as described above.

Mobile phone SO503i by NTT DoCoMo and Sony is used as the client. It is able to connect to the Internet and execute applications described with Java 2 Micro Edition Wireless SDK for DoJa[4]. This device has 120 pixel by 120 pixel LCD display. Figure 9 and 10 show the prototype system running on the actual device.

Debian/GNU Linux 3.0 with a self-made PC and Java 2 Runtime Environment 1.4 are used as the server.

HORB[5] and iHORB[6] are used as HTTP communication library. The former is a distribute object framework based on Java, the latter is an implementation of HORB for mobile environment.

\section{Conclusion}

This paper proposes a new approach for personal task schedule management based on decision-making theory.
Table 7. Scenario 2: Weighting vector of person by PageRank

\begin{tabular}{c|c} 
Name & Weight \\
\hline \hline A & $0.044(5)$ \\
W & $0.093(4)$ \\
X & $0.170(3)$ \\
Y & $0.370(1)$ \\
Z & $0.322(2)$
\end{tabular}

Table 8. Scenario 2: Evaluation value of alternatives

\begin{tabular}{l|c|c|c|c} 
Alternative & $N_{\text {task }}$ & $R_{\text {util }}$ & $R_{\text {pri. }}$ & $R_{\text {from }}$ \\
\hline \hline Schedule 1 & $5(1)$ & $0.500(6)$ & $19(1)$ & $1.049(1)$ \\
Schedule 2 & $4(2)$ & $0.851(2)$ & $16(3)$ & $0.956(2)$ \\
Schedule 3 & $4(2)$ & $0.979(1)$ & $14(5)$ & $0.879(3)$ \\
Schedule 4 & $4(2)$ & $0.809(4)$ & $15(4)$ & $0.678(5)$ \\
Schedule 5 & $4(2)$ & $0.830(3)$ & $17(2)$ & $0.727(4)$
\end{tabular}

Decision-making process of human consists of three types of activity, i.e., the intelligence activity, the design activity, and the choice activity. The proposed system can assist these three activities. Visualizer, a subsystem which supports the intelligence activity, i.e., indicates task condition of the user by chronogram-like display. It also shows overlapped tasks clearly. Optimizer, which helps the design activity, generates several alternative schedules with the active scheduler and the multi-objective genetic algorithm. These algorithms eliminate redundant candidates by comparisons with multiple objective functions. Recommender supports the choice activity using the analytic hierarchy process. It can evaluate qualitative and quantitative data at the same time easily. Additionally the system is able to analyze the human relationship of the user group with Analyzer which runs bsed on the PageRank algorithm.

We verify the function of the proposed system along with two scenarios. In the first scenario, we show how to resolve

Table 9. Scenario 2: Final result

\begin{tabular}{c|c} 
Alternative & Evaluation value \\
\hline \hline Schedule 1 & $0.194(4)$ \\
Schedule 2 & $0.207(1)$ \\
Schedule 3 & $0.206(2)$ \\
Schedule 4 & $0.189(5)$ \\
Schedule 5 & $0.203(3)$
\end{tabular}


overlap of tasks and set out the candidate schedules. In the second scenario, we establish the process that the priority of client is calculated and propagated.

A further direction of this study is to sophisticate our system. We are planning to perform an experiment with large-scale groups. In addition to the experiment we will investigate nature of personal tasks.

\section{References}

[1] C. A. Coello. An updated survey of evolutionary multiobjective optimization techniques: State of the art and future trends. Proc. of Congress on Evolutionary Computation, 1999.

[2] D.E.Goldberg. Genetic Algorithms in Search Optimization and Machine Learning. Addison-Wesley, 1989.

[3] M. Klusch. Information agent technology for the internet: A survey. Data and Knowledge Engineering, 36, 2001.

[4] I. NTT DoCoMo. i-mode java content developer's guide functional descriptions - release1.1. 2001.

[5] T. N. I. of Advanced Industrial Science and Technology. Horb. http://www.horb.org/, 2001.

[6] O. U. of Science. ihorb. http://mascot.mis.ous.ac.jp/horbous/test/, 2001

[7] L. Page, S. Brin, R. Motwani, and T. Winograd. The pagerank citation ranking: Bringing order to the web. Technical report, Stanford University, 1998.

[8] T. L. Saaty. Absolute and relative measurement with the ahp. Socio-Economic Planning Sciences, 20(6):327-331, 1986.

[9] T. L. Saaty. How to make a decision: The analytic hierarchy process. European Journal of Operational Research, 48(1):9-26, 1990.

[10] H. A. Simon. The New Science of Management Decision. Prentice-Hall Inc., 1977.

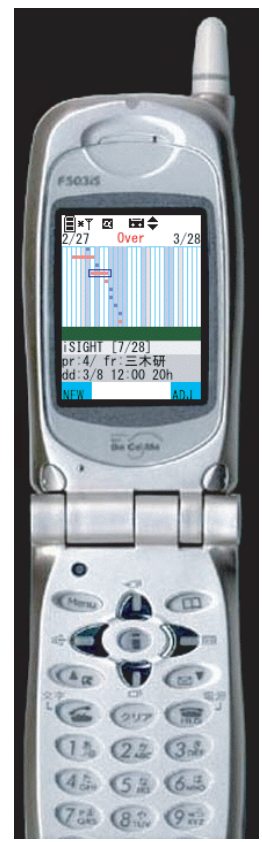

Figure 9. Initial condition on the long-term scale

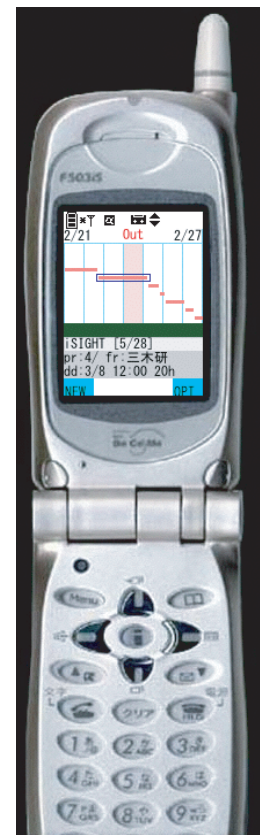

Figure 10. Proposed schedule by the system 